\title{
Constraining the difference in convective blueshift between the components of $\alpha$ Centauri with precise radial velocities
}

\author{
D. Pourbaix ${ }^{1,11, \star}$, D. Nidever ${ }^{2}$, C. McCarthy $^{3}$, R. P. Butler ${ }^{3}$, C. G. Tinney ${ }^{4}$, G. W. Marcy ${ }^{5}$, \\ H. R. A. Jones ${ }^{6}$, A. J. Penny ${ }^{7}$, B. D. Carter ${ }^{8}$, F. Bouchy ${ }^{9}$, F. Pepe ${ }^{9}$, J. B. Hearnshaw ${ }^{10}$, \\ J. Skuljan ${ }^{10}$, D. Ramm ${ }^{10}$, and D. Kent ${ }^{10}$ \\ ${ }^{1}$ Institut d'Astronomie et d'Astrophysique, Université Libre de Bruxelles, C.P. 226, Boulevard du Triomphe, \\ 1050 Bruxelles, Belgium \\ 2 Department of Physics and Astronomy, San Francisco State University, CA 94132, USA \\ 3 Department of Terrestrial Magnetism, Carnegie Institution of Washington, 5241 Broad Branch Road NW, \\ Washington DC 20015-1305, USA \\ 4 Anglo-Australian Observatory, PO Box 296, Epping, NSW 1710, Australia \\ 5 Department of Astronomy, University of California, Berkeley, CA 94720, USA \\ 6 Astrophysics Research Institute, Liverpool John Moores University, Twelve Quays House, \\ Egerton Wharf Birkenhead CH41 1LD, UK \\ 7 Rutherford Appleton Laboratory, Chilton, Didcot, Oxon, OX11 0QX, UK \\ 8 Faculty of Sciences, University of Southern Queensland, Toowoomba, 4350, Australia \\ 9 Observatoire de Genève, 1290 Sauvergny, Switzerland \\ 10 Mount John University Observatory, Department of Physics and Astronomy, University of Canterbury, \\ Orivate Bag 4800, Christchurch, New Zealand \\ 11 Department of Astrophysical Sciences, Princeton University, Princeton NJ 08544-1001, USA
}

Received 13 December 2001 / Accepted 20 February 2002

\begin{abstract}
New radial velocities of $\alpha$ Cen A \& B obtained in the framework the Anglo-Australian Planet Search programme as well as in the CORALIE programme are added to those by Endl et al. (2001) to improve the precision of the orbital parameters. The resulting masses are $1.105 \pm 0.0070 M_{\odot}$ and $0.934 \pm 0.0061 M_{\odot}$ for A and B respectively. The factors limiting how accurately these masses can be derived from a combined visual-spectroscopic solution are investigated. The total effect of the convective blueshift and the gravitational redshift is also investigated and estimated to differ by $215 \pm 8 \mathrm{~m} \mathrm{~s}^{-1}$ between the components. This suggests that the difference in convective blueshift between the components is much smaller than predicted from current hydrodynamical model atmosphere calculations.
\end{abstract}

Key words. stars: binaries: general - stars: individual: $\alpha$ Cen - methods: data analysis

\section{Introduction}

Pourbaix (1999) derived an orbit of $\alpha$ Cen A \& B whose main purpose was to illustrate how efficient the combination of visual and spectroscopic data can be in terms of distance and mass determination (Pourbaix 1998). This system also offers a laboratory for several areas of astronomy ranging from stellar evolution (Pourbaix et al. 1999; Guenther \& Demarque 2000; Morel et al. 2000) and asteroseismology (Bouchy \& Carrier 2001) to extra-solar planet search (Murdoch et al. 1993; Endl et al. 2001).

Instead of assuming an orbit and looking for a tiny variation caused by an unseen companion in some highly precise radial velocities, we use the data to improve the

\footnotetext{
Send offprint requests to: D. Pourbaix,

e-mail: pourbaix@astro.ulb.ac.be

* Post-doctoral Researcher, F. N. R. S., Belgium.
}

orbital parameters. The limits of such an approach are discussed in Sect. 2. We improve the spectro-visual orbit in Sect. 3 by combining the published data with new ones from the Anglo-Australian (Butler et al. 2001) and CORALIE (Queloz 2001) planet search projects. The corrections applied to these data are interpreted in Sect. 4 in terms of convective blueshift and gravitational redshift.

\section{The parallax as a model parameter}

$\alpha$ Cen is so close to us that there are several sources for the different ingredients required to derive the individual masses, for instance the mass sum and the mass ratio. We have already argued (Pourbaix 1998) that doublelined spectroscopic visual binaries offer a hypothesisfree determination of the distance and individual masses. The discrepancy between the former orbital parallax 


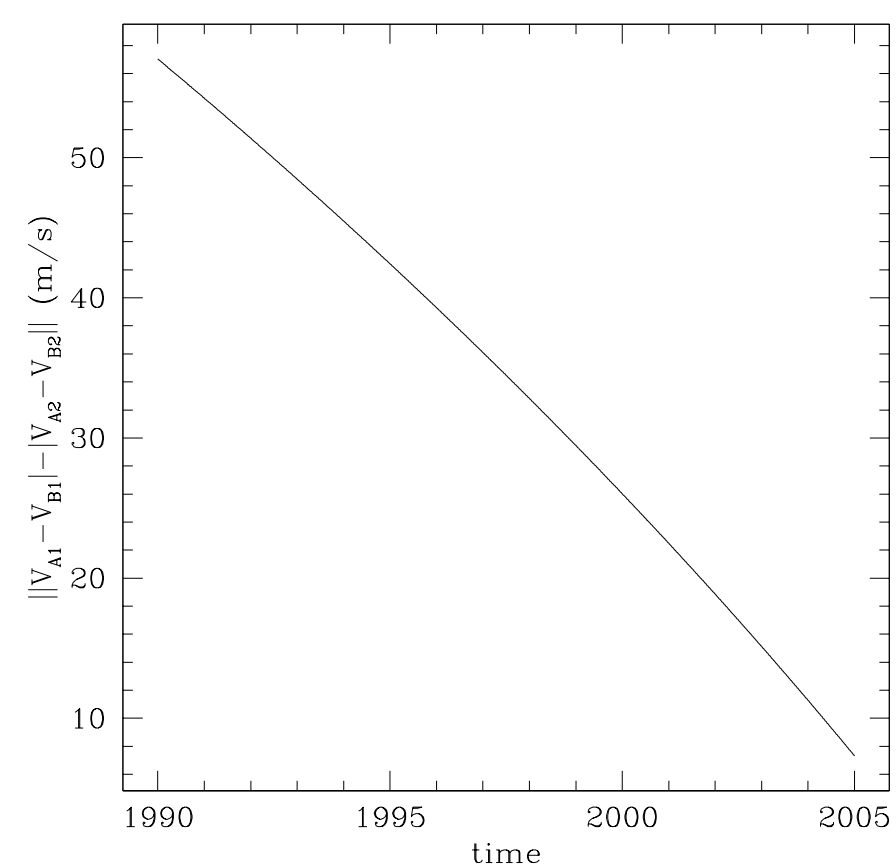

Fig. 1. Difference of the radial velocity difference between the two components derived from two parallaxes: 747.1 mas (Söderhjelm 1999) and 737 mas (Pourbaix et al. 1999). $V_{\mathrm{A} 1} \&$ $V_{\mathrm{B} 1}\left(\right.$ resp. $V_{\mathrm{A} 2} \& V_{\mathrm{B} 2}$ ) are the computed radial velocities from the orbit after Pourbaix et al. (1999) adopting 737 mas (resp. 747.1 mas) for the parallax.

$737.0 \pm 2.6$ mas (Pourbaix et al. 1999) and several others $742 \pm 1.42$ mas (HIP 71683, ESA 1997), 747.1 \pm 1.2 mas (Söderhjelm 1999) and the consequences on the masses are therefore rather puzzling (Guenther \& Demarque 2000).

Even if the old radial velocities exhibit a large scatter (the left end of the window in Fig. 2), in 1999, the precision of $K_{\mathrm{A}}$ and $K_{\mathrm{B}}$ (the amplitude of the radial velocity curves of A and B respectively) was better than $130 \mathrm{~m} \mathrm{~s}^{-1}$ for both components thanks to the sole data of Murdoch et al. (1993). But how accurate was the latter set? Pourbaix (1999) adopted their radial velocity zero point and shifted the earlier sets accordingly. With the semi-major axis of the visual orbit and the mass-ratio set, parallaxes of 737 mas and 750 mas, i.e. a change of $1.76 \%$, are distinguishable if $K_{\mathrm{A}}+K_{\mathrm{B}}$ is known to better than $170 \mathrm{~ms}^{-1}$. Unfortunately, the accuracy quoted by Murdoch \& Hearnshaw (1993) was only $200 \mathrm{~ms}^{-1}$.

The difference between $K_{\mathrm{A}}+K_{\mathrm{B}}$ derived with the $747.1 \pm 1.2$ mas (Söderhjelm 1999) and 737 mas is $169 \mathrm{~ms}^{-1}$. However, the closer one gets to the systemic velocity (Sep. 2006), the smaller the difference in the relative velocity (Fig. 1). Over the recent observing campaigns (about 5 years long), the effect of this parallax difference was too small to be detected even if accurate absolute radial velocities had been measured.

With the very precise relative radial velocities available now (Sect. 3), one can no longer consider the parallax as an independent parameter of the model. Indeed, the orbital parallax depends on the corrections applied to these velocities to change them into absolute ones. Therefore, either one assumes these shifts to be known and derives an orbital parallax or one assumes the latter and derives the former. From now on, we will assume that the parallax is known and set as 747.1 \pm 1.2 mas (Söderhjelm 1999).

\section{New data and orbital solution}

\subsection{AAT data}

The relative velocities of $\mathrm{A}$ and $\mathrm{B}$ were calculated in the usual way (Butler et al. 2001), and have typical internal measurement errors of $3 \mathrm{~ms}^{-1}$. The rms to a linear fit for $\mathrm{A}$ and $\mathrm{B}$ velocities is 5.8 and $3.6 \mathrm{~m} \mathrm{~s}^{-1}$. The absolute velocity zero-point was then derived by reanalyzing the A and B observations using the NSO solar atlas (Kurucz et al. 1984) as the stellar template (Nidever et al. 2002).

These two sets of radial velocities are absolute in some sense since $V_{\mathrm{A}}$ and $V_{\mathrm{B}}$ have the same zero point. However, neither the AAT nor the CORALIE data are absolute per se since they only barely account for the gravitational redshift and the convective blueshift. Although these two shifts are rather tough to derive, their total effect can be guessed.

Owing to the similarity between the template and component $\mathrm{A}$, the radial velocities of the latter were assumed to be absolute. The required correction to the velocities of B was $-215 \pm 8 \mathrm{~m} \mathrm{~s}^{-1}$ (presumably to account for the gravitational redshift, the convective blueshift and the template mismatch) in order for the orbital parallax to match the adopted value. This correction has a linear effect on the orbital parallax. Therefore, the $8 \mathrm{~ms}^{-1}$ uncertainty corresponds to the 1.2 mas uncertainty of the parallax after Söderhjelm (1999).

\subsection{CORALIE data}

The CORALIE data were obtained in the usual way, i.e. a synthetic K0V template was used for both A (slightly evolved G2V) and B (K1V). The rms to a linear fit for A and $\mathrm{B}$ velocities is 3.15 and $2.54 \mathrm{~m} \mathrm{~s}^{-1}$. These data together with the visual observations lead to a parallax of 694 mas, about $7 \%$ too small with a mass ratio also discrepant with respect to previous estimates (Kamper \& Wesselink 1978; Pourbaix et al. 1999). By adding corrections of $+9 \mathrm{~ms}^{-1}$ and $-215 \mathrm{~ms}^{-1}$ to the CORALIE data of $\mathrm{A}$ and $\mathrm{B}$ respectively, the parallax becomes consistent with the estimate after Söderhjelm (1999). A shift of $+40 \mathrm{~m} \mathrm{~s}^{-1}$ was also needed to put the CORALIE data in the same frame as the AAT.

We note that for component A the corrections required for the AAT and CORALIE velocities differ by $9 \mathrm{~ms}^{-1}$. This difference of $9 \mathrm{~m} \mathrm{~s}^{-1}$ for A could be caused by the use, in the CORALIE analysis, of the KOV template for the G2V star. The resulting spectral-type mismatch apparently causes a zero-point error of $9 \mathrm{~m} \mathrm{~s}^{-1}$ in A. Actually, although these $9 \mathrm{~m} \mathrm{~s}^{-1}$ are real, they are likely to be spread over the two components, caused by a template mismatch for the two components for both AAT and CORALIE. 


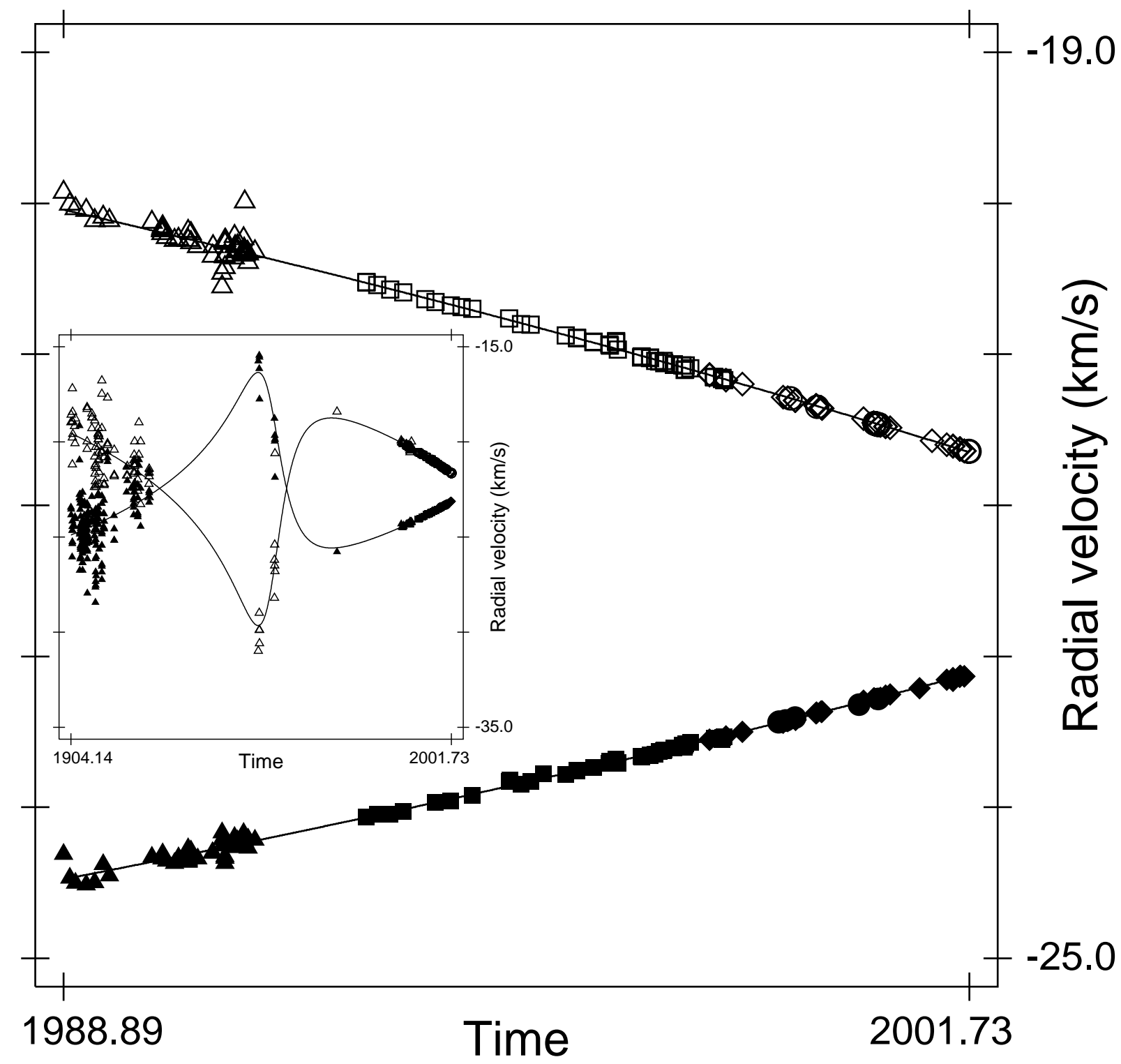

Fig. 2. Plot of the orbit from Table 1 and all the radial velocities obtained over the past 15 years. The five groups of very precise data are those of Murdoch et al. (1993) (triangles), Endl et al. (2001) (squares), the AAT (diamonds) and CORALIE (circles) ones. The window shows the orbit with all the existing radial velocities.

Hence, although the instruments, calibration, and reduction techniques are totally different, the AAT and CORALIE velocity sets both suggest that the sum of gravitational redshift and convective blueshifts are different by $\sim 220 \mathrm{~m} \mathrm{~s}^{-1}$ between A and B. We consider this difference of $\sim 220 \mathrm{~m} \mathrm{~s}^{-1}$ to be a significant measurement, albeit differential, of the effects of gravitational redshift and convective blueshift in G2V and K1V stars.

\subsection{Simultaneous solution}

The radial velocities published by Endl et al. (2001) are relative radial velocities. Therefore, the only information they constrain is the mass ratio (Eq. (2)). Once the absolute scale is set, the relative radial velocities can be adjusted accordingly. The whole set of spectroscopic data is thus composed of the data after Pourbaix et al. (1999), by Endl et al. (2001) and those in Table 2. A differential correction of $-43 \mathrm{~m} \mathrm{~s}^{-1}$ was also added to the data of B after Murdoch et al. (1993). This correction is well within the accuracy limits quoted by these authors. The zero point of these radial velocities was shifted by $-568 \mathrm{~m} \mathrm{~s}^{-1}$ to coincide with the AAT one. Consequently, all the older radial velocities were also shifted by $-568 \mathrm{~m} \mathrm{~s}^{-1}$ since they were initially tied (Pourbaix 1999) to the data of Murdoch et al. (1993). However, the relative weight of these latter data sets is so small with respect to the AAT and CORALIE ones that the effect of these changes is barely noticeable.

The relative position of the secondary has also been measured a few times over the past 15 years. These observations have been added to the Washington Double 
Table 1. Orbital parameters and their standard deviations. The parallax is adopted from Söderhjelm (1999).

\begin{tabular}{lcl}
\hline \hline Element & Value \\
\hline$a\left(^{\prime \prime}\right)$ & $17.57 \pm 0.022$ & \\
$i\left(^{\circ}\right)$ & $79.20 \pm 0.041$ & \\
$\omega\left(^{\circ}\right)$ & $231.65 \pm 0.076$ & \\
$\Omega\left(^{\circ}\right)$ & $204.85 \pm 0.084$ & \\
$e$ & $0.5179 \pm 0.00076$ & \\
$P($ yr $)$ & $79.91 \pm 0.011$ & \\
$T($ Besselian year $)$ & $1875.66 \pm 0.012$ & \\
$V_{0}\left(\mathrm{~km} \mathrm{~s}^{-1}\right)$ & $-22.445 \pm 0.0021$ & \\
$\varpi(\mathrm{mas}$ & $747.1 \pm 1.2$ & (adopted) \\
$\kappa=M_{\mathrm{B}} /\left(M_{\mathrm{A}}+M_{\mathrm{B}}\right)$ & $0.4581 \pm 0.00098$ & \\
$M_{\mathrm{A}}\left(M_{\odot}\right)$ & $1.105 \pm 0.0070$ & \\
$M_{\mathrm{B}}\left(M_{\odot}\right)$ & $0.934 \pm 0.0061$ & \\
\hline
\end{tabular}

Star Catalog of Observations and kindly supplied to us (Hartkopf \& Mason, priv. comm.). These data combined with all the radial velocities yield the orbit in Table 1.

Although the system exhibits a large proper motion $\left(3.7^{\prime \prime} / \mathrm{yr}\right.$ (ESA 1997)), our model does not account for any perspective effect. Although the visual observations cover more than three revolutions, they are not precise enough $\left(\sigma_{\theta}=2.3 \mathrm{deg}\right.$ and $\left.\sigma_{\rho}=0.6^{\prime \prime}\right)$ to exhibit any noticeable trend. Even if such a perspective effect should be accounted for at the level of precision for the modern radial velocities, the time interval they cover is too short for any noticeable effect.

With respect to the 1999 solution, one notes an upward revision of the fractional mass (still consistent with the estimate after Murdoch \& Hearnshaw 1993) that is hypothesis-free and relies on the relative velocities only (Sect. 4). This change combined with the larger parallax yield smaller masses, with a larger effect on A than on B. The precision on the masses $(<1 \%)$, about 4.5 times better than in 1999, therefore reflects the precision of the parallax and the accuracy of the mass ratio, orbital period and semi-major axis of the visual orbit. The revised mass of the primary agrees more with what was assumed in some recent theoretical investigations (e.g. Guenther $\&$ Demarque 2000). It is also consistent with the recent results of asteroseismology by Carrier et al. (2001).

With respect to the orbit, the radial velocities of Endl et al. (2001) have standard deviations of $11.9 \mathrm{~m} \mathrm{~s}^{-1}$ and $12.4 \mathrm{~m} \mathrm{~s}^{-1}$ for $\mathrm{A}$ and $\mathrm{B}$ respectively, consistent with the mean measurement errors $\left(12.3 \mathrm{~m} \mathrm{~s}^{-1}\right.$ and $\left.9.9 \mathrm{~m} \mathrm{~s}^{-1}\right)$. For the AAT data, the standard deviations are $4.8 \mathrm{~m} \mathrm{~s}^{-1}$ and $3.7 \mathrm{~m} \mathrm{~s}^{-1}$, again consistent with the estimated mean measurement errors. The CORALIE radial velocities have standard deviations of 7.6 and $4.3 \mathrm{~m} \mathrm{~s}^{-1}$ for A and B respectively. For this latter set, the larger noise on component $\mathrm{A}$ is due to some guiding problems noticed for bright stars.

\section{Blue and red shifts}

Radial velocities of double lined spectroscopic binaries are often used to derive the mass ratio (Irwin 1973). As long as the precision of these velocities does not exceed their accuracy, this is indeed a licit approach. Nowadays, the radial velocities have a precision better than $10 \mathrm{~ms}^{-1}$, however there has been little concern since Murdoch et al. (1993) about improving their accuracy. Indeed, whereas the precision is essential for the planet search campaign, very few people seem to worry about the accuracy (Griffin 1999; Gullberg 1999).

Starting with the equations of the radial velocity curves (Binnendijk 1960),

$$
\begin{aligned}
& V_{\mathrm{A}}=V_{0}-K_{\mathrm{A}}(\cos (\omega+v)+e \cos \omega), \\
& V_{\mathrm{B}}=V_{0}+K_{\mathrm{B}}(\cos (\omega+v)+e \cos \omega)
\end{aligned}
$$

where $v$ is the true anomaly and $\omega$ is the argument of the periastron of $\mathrm{B}$, one usually derives

$\frac{V_{\mathrm{B}}-V_{0}}{V_{0}-V_{\mathrm{A}}}=\frac{K_{\mathrm{B}}}{K_{\mathrm{A}}}=\frac{M_{\mathrm{A}}}{M_{\mathrm{B}}}=\frac{1}{\kappa}-1$

where $V_{\mathrm{A}}$ and $V_{\mathrm{B}}$ represent the absolute radial velocities of both components, not the observed ones. Indeed, the latter are affected by the gravitational redshift (Harwit 1998) and the convective blueshift (Dravins 1999). Therefore, unless the measured radial velocities are corrected for the total effect of these two shifts, these velocities do not yield the mass ratio.

If the mass and radius of the star are known, the gravitational redshift can be computed (Harwit 1998). Even at a $10 \mathrm{~ms}^{-1}$ precision, the differential effect of that shift would already be rather large. Thus, with the stellar parameters adopted below, the differential redshift is $143 \mathrm{~m} \mathrm{~s}^{-1}$, i.e. a $14 \sigma$ effect. Accounting for the convective blueshift is much more difficult because that would require some a priori understanding of the internal structure of the star.

Actually, one does not need (and should even avoid) the absolute radial velocities to derive the mass ratio. Indeed, the latter can already be obtained from the relative radial velocities. From

$V_{\mathrm{A}}=V_{0}+V_{0 \mathrm{~A}}+\Delta_{\mathrm{A}}$

$V_{\mathrm{B}}=V_{0}+V_{0 \mathrm{~B}}+\Delta_{\mathrm{B}}$

where $V_{0 \mathrm{~A}}$ and $V_{0 \mathrm{~B}}$ account for the zero points and the gravitational and convective shifts and $\Delta_{\mathrm{A}}$ and $\Delta_{\mathrm{B}}$ are the relative radial velocities, one derives

$\kappa=\frac{M_{\mathrm{B}}}{M_{\mathrm{A}}+M_{\mathrm{B}}}=\frac{\Delta_{\mathrm{A}}}{\Delta_{\mathrm{A}}-\Delta_{\mathrm{B}}}$.

Thus, from Endl et al. (2001), an orthogonal least-square fit yields $\kappa=0.457$ which is consistent with $0.454 \pm 0.002$ after Kamper \& Wesselink (1978).

We showed in the previous section that both the CORALIE and AAT data had to be differentially corrected by about $220 \mathrm{~m} \mathrm{~s}^{-1}$ in order to obtain the likely parallax and mass ratio. We can therefore write

$\left(G_{\mathrm{B}}-C_{\mathrm{B}}\right)-\left(G_{\mathrm{A}}-C_{\mathrm{A}}\right)=215 \pm 8 \mathrm{~ms}^{-1}$ 
Table 2. Relative and semi-absolute radial velocities.

\begin{tabular}{|c|c|c|c|c|c|c|c|c|c|}
\hline $\begin{array}{l}\text { JD } \\
-2400000\end{array}$ & $\begin{array}{r}\Delta_{\mathrm{A}} \\
\left(\mathrm{ms}^{-1}\right)\end{array}$ & $\begin{array}{r}\sigma_{\Delta_{\mathrm{A}}} \\
\left(\mathrm{ms}^{-1}\right)\end{array}$ & $\begin{array}{r}\Delta_{\mathrm{B}} \\
\left(\mathrm{ms}^{-1}\right)\end{array}$ & $\begin{array}{r}\sigma_{\Delta_{\mathrm{B}}} \\
\left(\mathrm{ms}^{-1}\right)\end{array}$ & $\left(\mathrm{ms}^{-1}\right) \stackrel{\stackrel{\circ}{V_{\mathrm{A}}}}{ }$ & $\begin{array}{r}\sigma_{V_{\mathrm{A}}} \\
\left(\mathrm{ms}^{-1}\right)\end{array}$ & $\left(\mathrm{ms}^{-1}\right) \begin{array}{r}V_{\mathrm{B}} \\
\end{array}$ & $\begin{array}{r}\sigma_{V_{\mathrm{B}}} \\
\left(\mathrm{ms}^{-1}\right)\end{array}$ & Reference \\
\hline 50831.27 & -339.6 & 2.1 & 224.7 & 1.7 & -23552.3 & 2.1 & -20920.2 & 1.7 & AAT \\
\hline 50833.24 & -338.0 & 2.4 & 223.0 & 2.0 & -23550.7 & 2.4 & -20921.9 & 2.0 & AAT \\
\hline 50917.12 & -317.5 & 3.9 & 188.0 & 4.4 & -23530.2 & 3.9 & -20956.9 & 4.4 & AAT \\
\hline 51002.93 & -287.8 & 3.0 & 163.8 & 2.9 & -23500.5 & 3.0 & -20981.1 & 2.9 & AAT \\
\hline 51189.88 & & & & & -23487.1 & $\leq 2$ & & & CORALIE \\
\hline 51193.89 & & & & & -23483.5 & $\leq 2$ & & & CORALIE \\
\hline 51213.28 & -228.2 & 2.6 & 77.3 & 3.2 & -23440.9 & 2.6 & -21067.6 & 3.2 & $\mathrm{AAT}$ \\
\hline 51223.87 & & & & & -23474.2 & $\leq 2$ & & & CORALIE \\
\hline 51236.29 & -214.7 & 3.7 & 73.4 & 3.0 & -23427.4 & 3.7 & -21071.5 & 3.0 & $\mathrm{AAT}$ \\
\hline 51251.86 & & & & & & & -21115.0 & $\leq 2$ & CORALIE \\
\hline 51274.23 & & & 54.5 & 3.3 & & & -21090.5 & 3.3 & $\mathrm{AAT}$ \\
\hline 51274.75 & & & & & -23455.7 & $\leq 2$ & & & CORALIE \\
\hline 51276.08 & -209.3 & 3.3 & 54.7 & 3.2 & -23422.0 & 3.3 & -21090.2 & 3.2 & $\mathrm{AAT}$ \\
\hline 51382.86 & -163.5 & 3.1 & 21.0 & 2.8 & -23376.2 & 3.1 & -21123.9 & 2.8 & AAT \\
\hline 51383.86 & -162.4 & 3.0 & 18.6 & 3.0 & -23375.1 & 3.0 & -21126.3 & 3.0 & AAT \\
\hline 51385.85 & -162.1 & 3.3 & 14.7 & 3.1 & -23374.8 & 3.3 & -21130.2 & 3.1 & AAT \\
\hline 51386.84 & -159.0 & 2.9 & 20.8 & 3.0 & -23371.7 & 2.9 & -21124.1 & 3.0 & $\mathrm{AAT}$ \\
\hline 51387.47 & & & & & & & -21174.4 & $\leq 2$ & CORALIE \\
\hline 51410.86 & -153.1 & 2.3 & 7.4 & 3.9 & -23365.8 & 2.3 & -21137.5 & 3.9 & $\mathrm{AAT}$ \\
\hline 51412.85 & -156.8 & 3.8 & 5.0 & 3.0 & -23369.5 & 3.8 & -21139.9 & 3.0 & AAT \\
\hline 51413.85 & -152.1 & 4.3 & 0.0 & 4.3 & -23364.8 & 4.3 & -21144.9 & 4.3 & $\mathrm{AAT}$ \\
\hline 51606.87 & & & & & -23368.3 & $\leq 2$ & & & CORALIE \\
\hline 51630.31 & -81.5 & 2.1 & -67.5 & 3.2 & -23294.2 & 2.1 & -21212.4 & 3.2 & $\mathrm{AAT}$ \\
\hline 51683.01 & -67.2 & 3.7 & -93.7 & 3.2 & -23279.9 & 3.7 & -21238.6 & 3.2 & AAT \\
\hline 51684.09 & -64.9 & 3.7 & -99.4 & 2.9 & -23277.6 & 3.7 & -21244.3 & 2.9 & $\mathrm{AAT}$ \\
\hline 51686.57 & & & & & & & -21281.3 & $\leq 2$ & CORALIE \\
\hline 51687.74 & & & & & & & -21282.1 & $\leq 2$ & CORALIE \\
\hline 51688.67 & & & & & & & -21285.4 & $\leq 2$ & CORALIE \\
\hline 51698.46 & & & & & & & -21289.7 & $\leq 2$ & CORALIE \\
\hline 51703.47 & & & & & & & -21292.6 & $\leq 2$ & CORALIE \\
\hline 51704.47 & & & & & -23331.7 & $\leq 2$ & & & CORALIE \\
\hline 51704.48 & & & & & & & -21292.3 & $\leq 2$ & CORALIE \\
\hline 51707.47 & & & & & & & -21287.2 & $\leq 2$ & CORALIE \\
\hline 51717.83 & -63.4 & 4.2 & -111.0 & 3.7 & -23276.1 & 4.2 & -21255.9 & 3.7 & $\mathrm{AAT}$ \\
\hline 51742.88 & -49.7 & 2.2 & -117.9 & 2.8 & -23262.3 & 2.2 & -21262.8 & 2.8 & AAT \\
\hline 51743.83 & -46.3 & 3.2 & -117.7 & 3.0 & -23259.0 & 3.2 & -21262.6 & 3.0 & AAT \\
\hline 51766.87 & -39.8 & 2.2 & -127.1 & 2.7 & -23252.5 & 2.2 & -21272.0 & 2.7 & AAT \\
\hline 51919.28 & 2.0 & 0.6 & & & -23210.7 & 0.6 & & & AAT \\
\hline 51984.32 & & & -211.4 & 1.6 & & & -21356.3 & 1.6 & AAT \\
\hline 52061.01 & 58.7 & 3.8 & -239.2 & 3.6 & -23154.0 & 3.8 & -21384.1 & 3.6 & AAT \\
\hline 52091.93 & 51.5 & 4.0 & -249.7 & 3.5 & -23161.2 & 4.0 & -21394.6 & 3.5 & AAT \\
\hline 52093.00 & 69.8 & 3.7 & -247.8 & 3.3 & -23142.9 & 3.7 & -21392.7 & 3.3 & AAT \\
\hline 52126.89 & 79.0 & 1.8 & -269.4 & 2.8 & -23133.7 & 1.8 & -21414.4 & 2.8 & AAT \\
\hline 52127.91 & 77.8 & 2.7 & -265.4 & 3.1 & -23134.9 & 2.7 & -21410.3 & 3.1 & AAT \\
\hline 52129.02 & 79.8 & 3.9 & -270.2 & 3.4 & -23132.9 & 3.9 & -21415.1 & 3.4 & AAT \\
\hline 52129.96 & 82.1 & 3.4 & -265.9 & 3.1 & -23130.6 & 3.4 & -21410.8 & 3.1 & AAT \\
\hline 52150.94 & 81.6 & 2.8 & -280.0 & 2.8 & -23131.1 & 2.8 & -21425.0 & 2.8 & AAT \\
\hline 52151.85 & 81.9 & 2.5 & -278.6 & 2.0 & -23130.8 & 2.5 & -21423.5 & 2.0 & $\mathrm{AAT}$ \\
\hline 52171.49 & & & & & & & -21467.7 & $\leq 2$ & CORALIE \\
\hline 52173.50 & & & & & & & -21466.6 & $\leq 2$ & CORALIE \\
\hline 52177.49 & & & & & & & -21468.0 & $\leq 2$ & CORALIE \\
\hline
\end{tabular}


where $G$ denotes the gravitational redshift and $C$ the convective blueshift (both regarded as positive quantities). It is worth keeping in mind that the $8 \mathrm{~ms}^{-1}$ uncertainty is based on the AAT data, results from the uncertainty on the adopted parallax only and does not account for the possible effect of the template mismatch. With the effective temperatures from Neuforge-Verheecke \& Magain (1997), the luminosities from Guenther \& Demarque (2000) and the masses we obtain (Table 1), $G_{\mathrm{A}}=560 \pm 14 \mathrm{~ms}^{-1}$ and $G_{\mathrm{B}}=703 \pm 20 \mathrm{~ms}^{-1}$ thus yielding

$C_{\mathrm{A}}-C_{\mathrm{B}}=72 \pm 26 \mathrm{~ms}^{-1}$

for the overall spectrum. The difference in convective blueshifts between the components of this system for the Fe I line at $\lambda 520 \mathrm{~nm}$ is about $500 \mathrm{~m} \mathrm{~s}^{-1}$ (Dravins \& Nordlund 1990). Hence, for at least one of these two stars, the Fe I line does not seems to be a good indicator of the overall convective blueshift.

\section{Conclusion}

We have solved the 3D two-body problem to derive masses and their precisions. The advantage of this solution is that it is completely independent of theoretical considerations such as models of stellar structure and evolution. Therefore, unless the original observations (the relative positions or the radial velocities) become questionable, these masses should be considered as true observables and the parameters of the theoretical models adjusted accordingly (not the opposite). However, in the case of doublelined spectroscopic visual binaries, although the mass ratio is accurately derived, only the precision can be guaranteed for the individual masses.

We have studied in detail the case of $\alpha$ Cen A \& B. Thanks to the accurate estimate of the parallax (e.g. after Hipparcos) and the combination of precise radial velocities and positions, it is possible to constrain the total effect of the convective blueshift and the gravitational redshift.

Acknowledgements. We thank L. Lindegren, the referee, for his valuable suggestions. The Anglo-Australian Planet Search team would like to thank the Director of the AAO, B. Boyle, and the superb technical support of the AAT staff - in particular E. Penny, R. Patterson, D. Stafford, F. Freeman, S. Lee, J. Pogson and G. Schafer. We acknowledge the UK and Australian government support of the AAT through their PPARC and DETYA (HRAJ, CGT, AJP); NASA grant NAG5-8299, \& NSF grant AST95-20443 (GWM); NASA grant
NAG5-11094 (DP); NSF grant AST-9988987 (RPB) and Sun Microsystems.

\section{References}

Binnendijk, L. 1960, Properties of Double Stars (University of Pennsylvania Press)

Bouchy, F., \& Carrier, F. 2001, A\&A, 374, L5

Butler, R. P., Tinney, C. G., Marcy, G. W., et al. 2001, ApJ, 555,410

Carrier, F., Bouchy, F., Meynet, G., et al. 2001, in IAU Colloq. 185, ASP Conf. Ser. 259, ed. C. Aerts, T. Bedding, \& J. Christensen-Dalsgaard

Dravins, D. 1999, in Precise stellar radial velocities, IAU Colloq. 170, ASP Conf. Ser. 185, ed. J. B. Hearnshaw, \& C. D. Scarfe, 268

Dravins, D., \& Nordlund, A. 1990, A\&A, 228, 184

Endl, M., Kürster, M., Els, S., Hatzes, A. P., \& Cochran, W. D. 2001, A\&A, 374, 675

ESA. 1997, The Hipparcos and Tycho Catalogues (ESA SP1200)

Griffin, R. E. M. 1999, in Precise stellar radial velocities, IAU Colloq., 170, ASP Conf. Ser. 185, ed. J. B. Hearnshaw, \& C. D. Scarfe, 52

Guenther, D. B., \& Demarque, P. 2000, ApJ, 531, 503

Gullberg, D. 1999, in Precise stellar radial velocities, IAU Colloq., 170, ASP Conf. Ser. 185, ed. J. B. Hearnshaw, \& C. D. Scarfe, 58

Harwit, M. 1998, Astrophysical concepts, 3rd ed.

Hearnshaw, J. B., \& Scarfe, C. D., (eds.) 1999, Precise stellar radial velocities

Irwin, J. B. 1973, ApJ, 179, 241

Kamper, K. W., \& Wesselink, A. J. 1978, AJ, 83, 1653

Kurucz, R. L., Furenlid, I., Brault, J., \& Testerman, L. 1984, Solar flux atlas from 296 to $1300 \mathrm{~nm}$ (National Solar Observatory)

Morel, P., Provost, J., Lebreton, Y., Thévenin, F., \& Berthomieu, G. 2000, A\&A, 363, 675

Murdoch, K., \& Hearnshaw, J. B. 1993, Observatory, 113, 79

Murdoch, K. A., Hearnshaw, J. B., \& Clark, M. 1993, ApJ, 413,349

Neuforge-Verheecke, C., \& Magain, P. 1997, A\&A, 328, 261

Nidever, D., Marcy, G. W., Butler, R. P., Fischer, D. A., \& Vogt, S. S. 2002, ApJ, submitted

Pourbaix, D. 1998, A\&AS, 131, 377

Pourbaix, D. 1999, in Precise stellar radial velocities, IAU Colloq., 170, ASP Conf. Ser. 185, ed. J. B. Hearnshaw \& C. D. Scarfe, 340

Pourbaix, D., Neuforge-Verheecke, C., \& Noels, A. 1999, A\&A, 344,172

Queloz, D. 2001, in Cool stars, stellar systems and the Sun, ASP Conf. Ser. 223, ed. R. J. Garcia Lopez, R. Rebolo, \& M. R. Zapatero Osorio, 59

Söderhjelm, S. 1999, A\&A, 341, 121 